\title{
A positive move: proton therapy in Australia
}

\author{
Scott Penfold ${ }^{1,2}$
}

Published online: 29 January 2018

(c) Australasian College of Physical Scientists and Engineers in Medicine 2018

In the September 2012 issue of this journal, Juergen Meyer in his Guest Editorial "Proton therapy: decisions, decisions" considered the broad and challenging question of whether proton therapy could be considered a wise investment for cancer treatment [5]. Discussions concerning this topic usually centre around the large capital expenditure required and the lack of clinical evidence in the form of phase III randomized clinical trials (RCTs) supporting proton therapy. Meyer does not pass any firm judgements concerning the cost-benefit of proton therapy. Instead, he suggests that the onus is on us as clinical experts to support the establishment of proton therapy centres, as it is only with these centres that we are able to gather robust scientific evidence to answer the underlying question: when, if ever, is proton therapy really superior to $X$-ray therapy? So where do we stand at the beginning of 2018? Have we progressed since 2012?

While we still may not have arrived at definitive answers to the questions posed by Meyer in 2012, we have at least made a start on the journey. In terms of clinical evidence, there are now several phase III RCTs underway comparing proton therapy and X-ray therapy [6]. While many see this as an essential undertaking to support the expanded use of proton therapy, there are others that question the applicability of RCTs in determining the direction of technological development in radiotherapy. Because of the long-term follow-up required for many treatment related effects in radiotherapy, the results of clinical trials may not be known for several years after all recruited patients have completed treatment. By this time, the technology used in the clinical trial may have been superseded, rendering the findings largely irrelevant. Consider retrospective comparisons of treatments delivered with intensity modulated X-ray therapy (IMRT

Scott Penfold

Scott.Penfold@sa.gov.au

1 Department of Medical Physics, Royal Adelaide Hospital, Adelaide, SA 5000, Australia

2 Department of Physics, University of Adelaide, Adelaide, SA 5005, Australia or VMAT) with passively scattered proton therapy (PSPT). The degree of conformity made possible with newer proton pencil beam scanning (PBS) exceeds that of PSPT systems in many cases and as such comparisons of IMRT/VMAT and PSPT may be unrelated to a comparison of IMRT/VMAT and PBS. The next major advancement may mean a similar redundancy in comparing IMRT/VMAT and PBS. So what are the alternatives? Some suggest that in silico clinical trials may be used to determine which technology of the day is likely to result in the best outcome for the patient $[1,2,4]$. In such an approach, a patient receives comparative plans with state-of-the-art delivery systems with clinical outcomes predicted with radiobiological modelling. The treatment plan with the more favourable predicted outcome is then selected. While this is an exciting area of research, the uncertainties inherent in patient specific radiobiological modelling leave some doubting the reliability of such an approach.

In terms of the capital expenditure required for proton therapy, there has been a substantial increase in the number of turn-key solutions available on the market, with at least seven commercial proton therapy vendors offering cyclotron- or synchrotron-based systems. This is a buyer's market that will drive prices down for the consumer. Currently, however, the cost savings associated with extra market competition is being offset by the advanced ancillary equipment being offered by vendors. Nowadays, packages usually include some form of in-room volumetric imaging in the form of cone beam CT (CBCT) or CT-on-rails, magnetic spot scanning nozzle designs, and six-degrees-offreedom robotic couches. On the plus side, it is likely that the introduction of these components will lead to use of proton therapy for an increasing number of clinical indications, since they will likely allow for margin reductions and a more complete realization of the dose saving advantages of proton therapy. This in turn will improve the financial viability of proton therapy centres.

At least one question alluded to by Meyer has been put to rest; should (or will) Australasia invest in proton therapy? In May 2017, the Federal Budget allocated funding of \$68 million to support the establishment of Australia's first proton 
therapy facility at the South Australian Health and Medical Research Institute (SAHMRI). One month later, the South Australian State Government reconfirmed its commitment of $\$ 44$ million to support the establishment of a proton therapy facility to be housed in a second SAHMRI building. This investment in infrastructure reflects a changing industrial scene in the South Australian capital.

So the decision has been made: Australia will join the particle therapy community. And in my opinion, as the title suggests, this is a good decision. The proposed building for Australia's first facility will be located in Adelaide's new BioMed City located on the historic North Terrace of central Adelaide. The precinct includes the new Royal Adelaide Hospital (opened 2017), SAHMRI (opened 2013), SAHMRI 2 (to be built), the University of Adelaide Health and Medical Sciences Building (opened 2017) and the Uni SA Health Innovation Building (opening 2018). The facility will be known as the Australian Bragg Centre for Proton Therapy and Research. The title reflects Adelaide's association with the discovery of the Bragg peak. This phenomenon was first observed by Bragg in 1904 [3] while conducting work at the University of Adelaide, just a few hundred metres down the road from the proposed facility location.

Looking ahead, one might question how a single proton therapy service will be accessed in a sparsely populated country with a state-based model of healthcare such as Australia? While the geographical scale does not compare to Australia, an interesting nationwide service for proton therapy has been developed by the Swedish healthcare system. The Skandionkliniken in Uppsala is jointly owned by seven university hospitals of Sweden. The centre is built on the principle of "shared governance-distributed competence" [7]. In this model of operation, the treatment centre in Uppsala is purely for treatment and all referrals are made through a patient's local university hospital. This means that treatment planning and follow-up are performed in the patient's local clinic, ensuring a high level of engagement across the country. This approach is made possible by todays advanced information technology environment. The Australasian College of Physical Scientists and Engineers in Medicine (ACPSEM) is taking a step in this direction with the creation of a Particle Therapy Working Group (PTWG) that is mandated to include members from all state Branches. This distributed group will ensure that the expertise across the nation is utilized in the national proton therapy facility and fed back into the national experience pool. A similar initiative has already been established by the Royal Australian and New Zealand College of Radiologists (RANZCR).

While Adelaide may be the first site of light ion therapy in Australasia, it is unlikely to be the only one. Business cases are in preparation for centres in Sydney, Melbourne, Brisbane and potentially other cities as well. With the increasing image guidance becoming available with proton therapy systems and development of adaptive radiotherapy workflows, I predict that we will see an increased benefit of proton therapy and an associated increase in utilization. I look forward to reading the next proton therapy-related editorial in several years' time to see if my predictions were correct.

\section{References}

1. Austin AM, Douglass MJJ, Nguyen GT, Penfold SN (2017) A radiobiological Markov simulation tool for aiding decision making in proton therapy referral. Phys Med 44:72-82. https://doi. org/10.1016/j.ejmp.2017.11.013

2. Blanchard P, Wong AJ, Gunn GB, Garden AS, Mohamed ASR, Rosenthal DI, Crutison J, Wu R, Zhang X, Zhu XR, Mohan R, Amin MV, Fuller CD, Frank SJ (2016) Toward a model-based patient selection strategy for proton therapy: external validation of photon-derived normal tissue complication probability models in a head and neck proton therapy cohort. Radiother Oncol 121(3):381-386. https://doi.org/10.1016/j.radonc.2016.08.022

3. Brown A, Suit H (2004) The centenary of the discovery of the Bragg peak. Radiother Oncol 73(3):265-268. https://doi. org/10.1016/j.radonc.2004.09.008

4. Langendijk JA, Lambin P, De Ruysscher D, Widder J, Bos M, Verheij M (2013) Selection of patients for radiotherapy with protons aiming at reduction of side effects: the model-based approach. Radiother Oncol 107(3):267-273. https://doi.org/10.1016/j.radon c. 2013.05.007

5. Meyer J (2012) Proton therapy: decisions, decisions. Australas Phys Eng Sci Med 35(3):253-256. https://doi.org/10.1007/s1324 6-012-0160-2

6. Mishra MV, Aggarwal S, Bentzen SM, Knight N, Mehta MP, Regine WF (2017) Establishing evidence-based indications for proton therapy: an overview of current clinical trials. Int J Radiat Oncol Biol Phys 97(2):228-235. https://doi.org/10.1016/j.ijrob p.2016.10.045

7. Montelius A, Grusell E, Karlsson M, Mattsson O, Mattsson S, Nilsson P (2007). Plans for particle therapy in Sweden. In: Proceedings of the 18th international conference of cyclotrons and their applications 2007, pp 219-221 